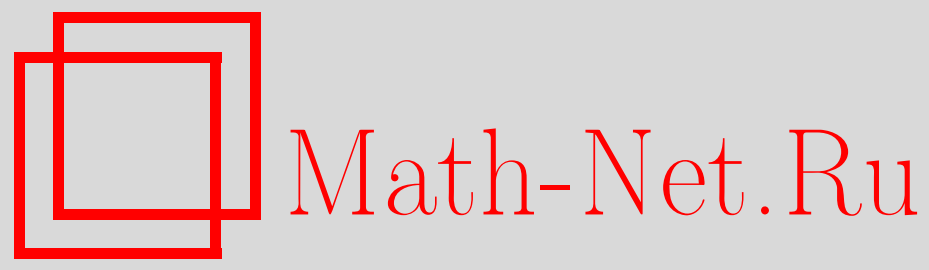

С. А. Степин, Об условиях конечности точечного спектра в несамосопряженной модели Фридрихса, Функи. анализ и его прил., 1997, том 31, выпуск 4, 83-86

DOI: https://doi.org/10.4213/faa500

Использование Общероссийского математического портала MathNet.Ru подразумевает, что вы прочитали и согласны с пользовательским соглашением http://www . mathnet.ru/rus/agreement

Параметры загрузки:

IP : 54.198 .67 .100

26 апреля 2023 г., 07:48:49

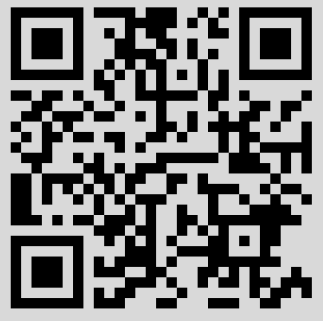


ПРЕДЛОЖЕНИЕ 2. (а) $\mathscr{D}(\pi) \subset H^{w}\left(X_{1}\right) \cap H^{w}\left(X_{2}\right)$;

(б) операторь $X_{1}, X_{2}$ определяют самосопряженное представление на $\mathscr{D}\left(\pi^{*}\right) \supset \mathscr{D}(\pi)$.

Определим функтор $F$ из категории $\mathscr{R}(\mathfrak{B})$ в категорию $\mathscr{R}(\mathfrak{A})$ следующим образом:

- если $\rho$ - представление *-алгебры $\mathfrak{B}, \rho(a)=A, \rho(b)=B$, то $F(\rho)=\pi^{*}$ $\left(\pi^{*}\right.$ - представление *-алгебры $\mathfrak{A}$ на $\mathscr{D}\left(\pi^{*}\right)$, порожденное операторами $\left.\left.X_{i}\right|_{\mathscr{D}\left(\pi^{*}\right)}, i=1,2\right)$;

- если $C: \mathscr{H}(\rho) \rightarrow \mathscr{H}(\hat{\rho}), C A=\widehat{A} C, C B=\widehat{B} C$, то

$$
F(C)=\mathscr{C}=\left(\begin{array}{ccc}
C & 0 & 0 \\
0 & C & 0 \\
0 & 0 & C
\end{array}\right) \otimes I_{L_{2}(\mathbb{R}, d x)} .
$$

ТЕОРЕМА 2. Функтор $F$ строгий и полньй.

\title{
ЛИТЕРАТУРА
}

1. Кругляк С. А., Самойленко Ю. С. Функц. анализ и его прил., 14, вып. 1, 60-62 (1980). 2. Пирятинская А. Ю., Самойленко Ю. С. Укр. матем. ж., 47, № 1, 70-78 (1995). 3. Kruglyak S., Piryatinskaya A. Preprint, Institut Mittag-Leffler (1996). 4. Schmüdgen $K$. Unbounded operator algebras and representation theory, AkademieVerlag, Berlin, 1990. 5. Nelson E. Ann. Math. 70, No. 3, 572-615 (1959). 6. Nizhnik L. P., Turowska L. B. Methods of Func. Anal. and Topol. В печати.

Институт математики НАН Украины, Киев

Поступило в редакцию 11 сентября 1996 г.

УДК 517.9

\section{Об условиях конечности точечного спектра в несамосопряженной модели Фридрихса ${ }^{\star}$}

\author{
(C) 1997. С. А. Степин
}

В работе изучается точечный спектр $\sigma_{p}$ операторов в $L_{2}(l), l=[a, b] \subset \mathbb{R}$, вида $H=H_{0}+V$, где $H_{0}$ - оператор умножения на независимую переменную, а возмущение $V$ - интегральный оператор с ядром $v$, удовлетворяющим некоторым условиям гладкости.

Известно (см. [1-3]), что спектральные свойства операторов самосопряженной модели Фридрихса существенно зависят от степени гёльдеровской гладкости ядра возмущения. В этих терминах гранищей конечности сингулярного спектра служит показатель гёльдеровости $1 / 2$. В несамосопряженном случае даже при условии бесконечной дифференцируемости ядра $v$ может происходить сгущение собственных значений к отрезку $l$; в [4] построен соответствующий пример и исследована структура множества точек накопления собственных значений.

\footnotetext{
* Работа выполнена при поддержке РФФИ (грант № 96-01-00333).
} 
Для однопараметрического семейства операторов $H(\alpha)=H_{0}+V(\alpha)$ специального вида в работе автора [5] предложен способ доказательства конечности точечного спектра, использующий вариацию параметра $\alpha$ и основанный на соображениях аналитической теории возмущений. Этот подход удается распространить на достаточно общую операторную ситуацию, оставаясь в рамках несамосопряженной модели Фридрихса. Отметим, в этой связи, что аналитическая теория возмущений, строго говоря, неприменима к собственным значениям, погруженным в непрерывный спектр; возникающие при этом эффекты изучались в [6].

Введем в рассмотрение класс $\mathfrak{h}^{\mu}, 0<\mu \leqslant 1$, функций $\varphi: l \rightarrow \mathbb{C}$, для которых

$$
\|\varphi\|_{\mu}:=\sup _{\omega \neq \omega^{\prime}}\left\{|\varphi(\omega)|+\frac{\left|\varphi(\omega)-\varphi\left(\omega^{\prime}\right)\right|}{\left|\omega-\omega^{\prime}\right|^{\mu}}\right\}<\infty .
$$

В $L_{2}(l)$ рассматривается однопараметрическое семейство операторов модели Фридрихса $H(\alpha)=H_{0}+V(\alpha)$, где $V(\alpha)$ - интегральный оператор с ядром $v\left(\omega, \omega^{\prime}, \alpha\right)$, а $\alpha$ - комплексный параметр; предполагается, что при фиксированном $\alpha$ ядро $v(\alpha): l \times l \rightarrow \mathbb{C}$ удовлетворяет следующим условиям:

$$
\begin{gathered}
\|v(\alpha)\|_{\mu}:=\sup _{\omega^{\prime} \in l}\left\|v\left(\cdot, \omega^{\prime}, \alpha\right)\right\|_{\mu}+\sup _{\omega \in l}\|v(\omega, \cdot, \alpha)\|_{\mu}<\infty, \quad \mu>0, \\
v\left(a, \omega^{\prime}, \alpha\right)=v\left(b, \omega^{\prime}, \alpha\right)=v(\omega, a, \alpha)=v(\omega, b, \alpha)=0 .
\end{gathered}
$$

Задача отыскания собственных значений оператора $H(\alpha)$, лежащих вне $l$, сводится к вопросу о разрешимости в $\mathfrak{h}^{\nu}, \nu>0$, уравнения

$$
\varphi(\omega)+K(\lambda, \alpha) \varphi(\omega)=0,
$$

где $K(\lambda, \alpha)=V(\alpha)\left(H_{0}-\lambda E\right)^{-1}$. Уравнение (3) естественно рассматривать для значений $\lambda$, принадлежащих комплексной плоскости с разрезом $l$, различая при этом края разреза; другими словами, область П изменения спектрального параметра $\lambda$ представляет собой поверхность с краем, полученную пополнением $\mathbb{C} \backslash l$ относительно метрики $\rho\left(\lambda, \lambda^{\prime}\right)$, определяемой как точная нижняя грань длин кривых, соединяющих $\lambda$ и $\lambda^{\prime}$ и не пересекающих отрезок $l$. Если $\lambda=$ $c \pm i 0 \in \partial \Pi$, то для $\varphi \in \mathfrak{h}^{\nu}, \nu>0$, имеем

$$
K(c \pm i 0, \alpha) \varphi(\omega)=\text { v.p. } \int_{l} \frac{v\left(\omega, \omega^{\prime}, \alpha\right) \varphi\left(\omega^{\prime}\right)}{\omega^{\prime}-c} d \omega^{\prime} \pm i \pi v(\omega, c, \alpha) \varphi(c) .
$$

Обозначим через $\Lambda(\alpha)$ множество значений параметра $\lambda \in \Pi$, при которых (3) имеет нетривиальное решение в классе $\mathfrak{h}^{\nu}, \nu>0$.

ПРеДЛОЖЕНИЕ (ср. [7]). Для каждого $\lambda \in \Pi$ и произвольного $\nu \in(0, \mu)$ оператор $K(\lambda, \alpha)$ компактен в $\mathfrak{h}^{\nu}$ и гёльдеровски непрерывно зависит от $\lambda \in \Pi$ в соответствующей операторной норме.

СлЕДСТВИЕ. Множество $\Lambda(\alpha) \subset \Pi$ замкнуто.

Учитывая тот факт, что $\sigma_{p}(H(\alpha)) \subset \Lambda(\alpha)$, приходим к следующему выводу: точки накопления собственных значений оператора $H(\alpha)$ принадлежат множеству $\Omega(\alpha):=\Lambda(\alpha) \cap \partial \Pi$.

Теорема 1. Пусть ядро $v\left(\omega, \omega^{\prime}, \alpha\right)$ удовлетворяет условиям (1)-(2) u, кроме того, аналитично по параметру а относительно нормьи $\|\cdot\|_{\mu}$ в некоторой комплексной окрестности $O\left(\alpha_{0}\right)$ точки $\alpha=\alpha_{0}$. Если множество 
$\bigcup_{\alpha \in O\left(\alpha_{0}\right)} \Omega(\alpha)$ конечно и $\bigcap_{\alpha \in O\left(\alpha_{0}\right)} \Omega(\alpha)=\varnothing$, то точечный спектр оператора $H\left(\alpha_{0}\right)$ конечен.

Допустим, что множество собственных значений оператора $H\left(\alpha_{0}\right)$ бесконечно и, таким образом, имеет точку сгущения $c \in l$; для определенности будем считать, что $c-$ внутренняя точка отрезка $l$ и накопление происходит из верхней полуплоскости так, что $c+i 0 \in \Omega\left(\alpha_{0}\right)$. Те значения $\alpha \in O\left(\alpha_{0}\right)$, для которых $c+i 0 \in \Omega(\alpha)$, будем называть особыми точками.

Операторы $K(c+i 0, \alpha), \alpha \in O\left(\alpha_{0}\right)$, компактны в $\mathfrak{h}^{\nu}, \nu \in(0, \mu)$, и образуют голоморфное семейство. Согласно известному результату аналитической теории возмущений (см. [8]), либо все $\alpha \in O\left(\alpha_{0}\right)$ суть особые точки, либо каждое компактное подмножество из $O\left(\alpha_{0}\right)$ содержит лишь конечное число особых точек. Поскольку $\bigcap_{\alpha \in O\left(\alpha_{0}\right)} \Omega(\alpha)=\varnothing$, то реализуется вторая возможность и, следовательно, найдется окрестность $\widetilde{O}\left(\alpha_{0}\right) \subset O\left(\alpha_{0}\right)$, не содержащая других особых точек, кроме $\alpha_{0}$. Ввиду конечности множества $\bigcup_{\alpha \in O\left(\alpha_{0}\right)} \Omega(\alpha)$ существует интервал $\Delta \subset \partial П$, такой, что

$$
\Omega\left(\alpha_{0}\right) \cap \Delta=\{c+i 0\} \quad \text { и } \underset{\alpha \in \widetilde{O}\left(\alpha_{0}\right), \alpha \neq \alpha_{0}}{\bigcup} \Omega(\alpha) \cap \Delta=\varnothing .
$$

Применение схемы доказательства теоремы 1 работы [5] с учетом сделанного наблюдения приводит к противоречию с предположением о бесконечности $\sigma_{p}\left(H\left(\alpha_{0}\right)\right)$.

В качестве приложения рассмотрим оператор модели Фридрихса в случае одномерного возмущения

$$
v\left(\omega, \omega^{\prime}, \alpha\right)=p(\omega) q\left(\omega^{\prime}, \alpha\right),
$$

где $p(\cdot), q(\cdot, \alpha) \in \mathfrak{h}^{\mu}, \mu>0, p(a)=p(b)=q(a, \alpha)=q(b, \alpha)=0$ и $v(\omega, \omega, \alpha) \in \mathbb{R}$, $\omega \in l$; кроме того, предполагается, что функция $q(\cdot, \alpha)$ аналитична по параметру $\alpha$ относительно нормы $\|\cdot\|_{\mu}$.

Необходимое условие разрешимости уравнения (3), отвечающего $\lambda=c \pm i 0$, имеет вид (см. [9])

$$
1+\mathrm{v} \cdot \mathrm{p} \cdot \int_{l} \frac{p(\omega) q(\omega, \alpha)}{\omega-c} d \omega \pm i \pi p(c) q(c, \alpha)=0,
$$

и, стало быть, $p(c) q(c, \alpha)=0$, когда $c+i 0$ или $c-i 0$ принадлежит $\Omega(\alpha)$. Таким образом, если для $\alpha=\alpha_{0}$ найдется окрестность $O\left(\alpha_{0}\right)$, такая, что множество $\left\{\omega \in l: p(\omega) q(\omega, \alpha)=0, \alpha \in O\left(\alpha_{0}\right)\right\}$ конечно и $\bigcap_{\alpha \in O\left(\alpha_{0}\right)} \Omega(\alpha)=\varnothing$, то конечен точечный спектр оператора $H\left(\alpha_{0}\right)$ с возмущением, задаваемым ядром (4).

Последнее реализуется, например, в случае, когда зависимость от параметра $\alpha$ в (4) линейная: $q(\omega, \alpha)=\alpha q(\omega)$; здесь в качестве $O\left(\alpha_{0}\right)$ надо взять область, содержащую точки $\alpha=0$ и $\alpha=\alpha_{0}$.

ТЕОРема 2. Пусть $p, q \in \mathfrak{h}^{\mu}, \mu>0, p(a)=p(b)=q(a)=q(b)=0$, причем $p(\omega) q(\omega)$ вещественнозначна и имеет конечное число нулей на $l=[a, b]$. Тогда точечныи спектр оператора $H=\omega \cdot+p(\omega)(\cdot, \bar{q})_{L_{2}(l)}$ cocmouт из конечного множества собственных значений.

Автор благодарит Р. А. Минлоса за полезные обсуждения и замечания. 


\section{ЛитеРАТУРА}

1. Фаддеев Л. Д. Труды МИАН, 73, 292-313 (1964). 2. Павлов Б. С., Пеmpac C. В. Функц. анализ и его прил., 4, вып. 2, 54-61 (1970). 3. Дынькин Е. М., Набоко С. Н., Яковлев С. И. Алгебра и анализ, 3, вып. 2, 77-90 (1991). 4. Набоко С. Н. Зап. науч. сем. ЛОМИ, 39, 40-58 (1974). 5. Степин С. А. Известия PAH, 60, № 6, 201-221 (1996). 6. Howland J. S. Pacif. J. Math., 55, 157-174 (1974). 7. Yafaev D. R. Mathematical scattering theory. Transl. Math. Monographs, Vol. 105, Am. Math. Soc., 1992. 8. Като T. Теория возмущений линейных операторов. Мир, М., 1972. 9. Фридрихс K. О. Возмущение спектра операторов в гильбертовом пространстве. Мир, М., 1969.

Московский государственный университет им. М. В. Ломоносова,

Поступило в редакцию механико-математический факультет

4 февраля 1997 г.

УдК 517.518

\section{Ортогональные всплески на локально компактных абелевых группах}

(C) 1997. Ю. А. ФАРКОВ

1. Пусть $G$ - локально компактная абелева группа, $\mu$ - мера Хаара на $G$, $L^{2}(G):=L^{2}(G, \mu), H$ - дискретная подгруппа в $G$, такая, что факторгруппа $G / H$ компактна, $A$ - автоморфизм группы $G$, такой, что $A(H)$ - собственная подгруппа в $H$, и $s:=\operatorname{card}(H / A(H))$. В предположении, что функция $\varphi \in$ $L^{2}(G)$ удовлетворяет уравнению

положим

$$
\varphi(g)=\sum_{h \in H} b(h) \varphi(A g-h), \quad b \in L^{2}(H), g \in G,
$$

$$
\begin{aligned}
V(\varphi) & :=\operatorname{clos}_{L^{2}(G)} \operatorname{span}\{\varphi(\cdot-h) \mid h \in H\}, \\
V_{j} & :=\left\{f\left(A^{j} \cdot\right) \mid f \in V(\varphi)\right\}, \quad j \in \mathbb{Z} .
\end{aligned}
$$

Из (1) следует, что $V_{j} \subset V_{j+1}$ для всех $j \in \mathbb{Z}$. Ортогональное дополнение к $V_{j}$ в $V_{j+1}$ обозначается через $W_{j}$.

ЗАДАчА А. Найти функции $\psi_{1}, \ldots, \psi_{s-1}$ в $L^{2}(G)$, такие, что $W_{0}$ является прямой суммой подпространств

$$
W_{0}^{(i)}:=\cos _{L^{2}(G)} \operatorname{span}\left\{\psi_{i}(\cdot-h) \mid h \in H\right\}, \quad i=1, \ldots, s-1 .
$$

Относительно способов решения этой задачи при построении всплесковых базисов в $L^{2}\left(\mathbb{R}^{d}\right)$ см., например, $[1,2]$. Пару $(G, A)$ будем называть допустимой, если 1) группа $G$ метризуема, причем выполняется вторая аксиома счетности, и 2) автоморфизм $A$ непрерывен, а его обратный $A^{-1}$ является сжатием. Некоторые примеры допустимых пар $(G, A)$ с $G \neq \mathbb{R}^{d}$ даны в [3]. 